\title{
Simplex-optimized Chromatographic Resolution of Selected Ionic Liquid Cations Utilizing a Polar Reversed-Phase System
}

\author{
Joanna Nichthauser,* Monika Paszkiewicz,* Andrzej C. SkŁadanowski,** and \\ Piotr STEPNOWSKI ${ }^{* \dagger}$
}

*Department of Environmental Analysis, Faculty of Chemistry, University of Gdańsk, Sobieskiego 18, PL-80-952 Gdańsk, Poland

**Department of Molecular Enzymology, Intercollegiate Faculty of Biotechnology, Medical University of Gdańsk, ul. Dębinki 1, 80-211 Gdańsk, Poland

\begin{abstract}
This study reports on optimization of the RP-HPLC separation of imidazolium and pyridinium ionic liquid cations using a variable-size simplex algorithm. Under the optimized conditions, all critical pairs of ionic liquids were successfully separated in a single chromatographic run. The mobile phase at the point corresponding to the optimum consisted of $10 \%$ $\mathrm{MeOH}$ and $90 \% 15 \mathrm{mM} \mathrm{KH}_{2} \mathrm{PO}_{4} / \mathrm{H}_{3} \mathrm{PO}_{4}$ with $\mathrm{pH} 3.43$. The coefficients of asymmetry for all of the compounds analyzed at the simplex algorithm optimum ranged from 0.83 to 2.91 .
\end{abstract}

(Received April 4, 2008; Accepted May 20, 2008; Published October 10, 2008)

\section{Introduction}

Room-temperature ionic liquids (RTIL) are a new class of compounds usually consisting of an organic cation and a variety of organic or inorganic anions. Apart from their negligible vapor pressure, believed to be the main "green" feature of ionic liquids, one of their most attractive characteristics is the potential to be "designed to order". Liquids below $100^{\circ} \mathrm{C}$, these compounds make ideal non-volatile solvents for a variety of industrial chemical syntheses. They are also applicable as biocatalytic media, as phases and additives in separation processes, or as alternative lubricants. RTIL already in common use typically involve nitrogen- or phosphorus-containing organic cations, such as alkylimidazolium, alkylpyridinium, alkylpyrrolidinium or alkylphosphonium, and anions, like bis(trifluoromethanesulfonyl)imide, hexafluorophosphate or tetrafluorophosphate. ${ }^{1}$

Any physicochemical studies of these chemicals require reliable, relatively simple and reproducible analytical techniques. Our group has recently developed a number of instrumental methods that are now ready for testing on various matrices containing ionic liquids. Selected 1-alkyl- and 1-aryl-3-methylimidazoliumbased ionic liquid cations were separated using reversed-phase high-performance liquid chromatography with electrospray ionization mass detection. ${ }^{2}$ Simple and selective, this method is thought to be applicable to numerous ionic liquids based on a similar design. A strong stationary phase cation exchanger was also initially applied to separate 1-alkyl-3-methylimidazolium cations and one 4-methyl- $N$-butylpyridinium cation. ${ }^{3,4}$ When using this phase, several interactions occurred with varying strength, depending on the mobile-phase composition. Capillary electrophoresis was also applied to resolve certain imidazolium ionic liquid cations in standard mixtures. ${ }^{5}$ Nevertheless, the

$\dagger$ To whom correspondence should be addressed.

E-mail: sox@chem.univ.gda.pl separation of ionic liquids in these modes is less selective, especially with respect to highly hydrophilic solutes. It can be improved, however, by invoking interactions between solutes and non-hydrophobic separation phases. A number of other interactions are also possible between an ionic liquid cation and a surface (dispersive, electrostatic, dipole-ion, ion-ion or $\pi \cdots \pi$. Our recent studies of the phenyl- and pentafluorophenylpropylbonded phases have demonstrated the potential usefulness of $\pi \cdots \pi$ interactions between solutes and stationary-phase ligands in the selective separation of imidazolium and pyridinium ionic liquid cations with extended $\pi$-electron systems. ${ }^{6,7}$ These methods, however, still need optimization with regard to the resolution of isomers, peak symmetry and time of analysis.

Sequential simplex methods are common and efficient optimization techniques applied in many fields of chemistry and chemical engineering. Simplex optimization can be used to experimentally determine optimum conditions, and also to improve the process efficiency, product quality or instrument performance..$^{8-11}$ A number of studies on the modification and improvement of the original algorithm have been published over the last 20 years. ${ }^{12-16}$

In this study, we therefore decided to utilize the simplex method for optimizing the mobile phase content in an analysis of ionic liquid cations. In this paper, we present our data on the optimization of HPLC separation of ionic liquid cations on a polar reverse stationary phase using a variable-size simplex algorithm. ${ }^{17}$

\section{Experimental}

\section{Reagents}

The ionic liquids selected for these studies were purchased from Merck KGaA (Darmstadt, Germany): 1-ethyl-3methylimidazolium (EMIM), 1-ethyl-3-ethylimidazolium (EEIM), 1-propyl-3-methylimidazolium (PMIM), 1-propyl-3ethylimidazolium (PEIM), 1-butyl-3-methylimidazolium 
(BMIM), 1-butyl-3-ethylimidazolium (BEIM), 1-hexyl-3methylimidazolium (HMIM), 1-benzyl-3-methylimidazolium (BzMIM), 1-ethylbenzyl-3-methylimidazolium (EBzMIM) and $\mathrm{N}$-butyl-4-methylpyridinium (MBPyr) chlorides. All ionic liquids were used as obtained, without additional pretreatment. Standard stock solutions $(1 \mathrm{mM})$ were prepared by dissolving a weighed amount of the compounds in distilled water. The monopotassium phosphate and $85 \%$ orthophosphoric acid used for the buffer were obtained from POCh (Gliwice, Poland); HPLC-gradient grade methanol was purchased from Scharlau Chemie (Barcelona, Spain).

\section{Apparatus, analysis and simplex calculations}

The analytical system used in the experiments was a highperformance liquid chromatograph (Perkin Elmer Series 200) consisting of a chromatographic interface (Link 600), binary pump, UV/Vis detector, vacuum degasser and Rheodyne injection valve. The ionic liquids were separated on a Gemini C-6 $150 \times 4.6 \mathrm{~mm}$ polar RP column (Phenomenex) packed with $5 \mu \mathrm{m}$ particles of pore size $110 \AA$

The optimization of the ionic liquid separation on the polar reversed phase was studied using multicompound analysis in the isocratic mode. The analyses were performed at ambient temperature at a flow rate of $0.8 \mathrm{ml} \mathrm{min}{ }^{-1}$, and the elution profiles were monitored at $\lambda=218 \mathrm{~nm}$. The separation column was equilibrated with the mobile phase until baseline stabilization, at which point sample injections $(10 \mu \mathrm{l})$ were made.

The mobile phase was methanol mixed with various proportions of phosphate buffer $\left(\mathrm{KH}_{2} \mathrm{PO}_{4} / \mathrm{H}_{3} \mathrm{PO}_{4}\right)$ at $\mathrm{pH} 3.0-$ 4.3. The retention factors, $k$, were calculated as $\left(t-t_{0}\right) / t_{0}$.

The simplex method was used to optimize the separation of standards of ten typical alkylimidazolium and alkylpyridinium ionic liquid cations. The separations were carried out under isocratic conditions; a variable-size simplex algorithm was applied.

\section{Results and Discussion}

A chemometric approach based on the simplex method was applied to optimize the separation of congeneric groups of typical alkylimidazolium and alkylpyridinium ionic liquids. Special attention was paid to short-chain hydrophilic cations, like EMIM, EEIM, PMIM, PEIM and BMIM; these entities have similar lipophilicities, which makes their separation difficult. When using a simplex, each vertex corresponds to a set of experimental conditions. The three variables expected to have a considerable effect on the chromatographic separation of the ionic liquids were the percentage of organic modifier $(\mathrm{MeOH})$, the concentration of the phosphate buffer, and the $\mathrm{pH}$ The initial simplex consisting of the first four vertices was established on the basis of earlier studies of the ionic liquid retention mechanism on chemically bonded stationary phases with different specific structural properties. . $^{2,4,6,7,18}$ The points of

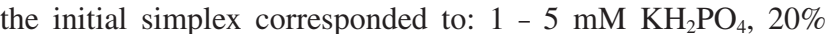
$\mathrm{MeOH}, \mathrm{pH} 3.0 ; 2$ - 15 mM KH $\mathrm{PO}_{4}, 10 \% \mathrm{MeOH}, \mathrm{pH} 3.5 ; 3-25$ $\mathrm{mM} \mathrm{KH} \mathrm{PO}_{4}, 3 \% \mathrm{MeOH}, \mathrm{pH} 4.0 ; 4-10 \mathrm{mM} \mathrm{KH} \mathrm{PO}_{4}, 15 \%$ $\mathrm{MeOH}, \mathrm{pH} 3.75$.

The separation quality of the analyzed ionic liquid cations for achieving the maximum resolution in the minimum assay time was assessed by calculating the value of the chromatographic response function (CRF) according the following equation: ${ }^{17}$

$$
\mathrm{CRF}=\sum_{\mathrm{i}=1}^{\mathrm{L}} R_{\mathrm{i}}+L^{\mathrm{a}}-b\left|T_{\mathrm{A}}-T_{\mathrm{L}}\right|+c\left(T_{1}-T_{\mathrm{o}}\right),
$$

where $R_{\mathrm{i}}$ is the resolution between adjacent peak pairs; this parameter was limited to a maximum value of 1.5 , so all pairs with $R_{\mathrm{i}}$ below this value made no contribution to CRF; $L$ is the number of peaks detected; $T_{\mathrm{A}}$ is the specified time of analysis ( 25 min in our work); $T_{\mathrm{L}}$ is the retention time of the last eluted peak; $T_{1}$ is the retention time of the first eluted peak; $T_{\mathrm{o}}$ is a specified minimum retention time ( $3 \mathrm{~min}$ ); $a, b, c$ are weighting factors ( $a=1, b=1$ and $c=1)$. The values of the weighting factors $(a, b$ and $c)$ were set in an arbitrary way by the authors. From the resulting chromatogram under each set of conditions, $\mathrm{CRF}$ was calculated and the relative responses ranked. The advantage of CRF for the simplex method is that it enables the weighting of important chromatographic features $\left(R_{\mathrm{i}}\right.$ and $\left.T_{\mathrm{A}}\right)$ for simplex movements that result in higher CRF values with increased resolution, a reasonably short analysis time $\left(T_{\mathrm{A}}-T_{\mathrm{L}}\right)$ and good retention $\left(T_{1}>T_{0}\right)$.

The peak shapes of all ionic liquid cations analyzed, evaluated using the coefficient of asymmetry $\left(f_{\mathrm{AS}}\right)$, were also a quality criterion of the chromatographic separation obtained. The peak asymmetry coefficient is the ratio (at $10 \%$ of the peak height) of the distance between the peak apex and the back side of the chromatographic curve to the distance between the peak apex and the front side of the chromatographic curve. A value $>1$ is a tailing peak, while a value $<1$ is a fronting peak.

The experiments described by the initial simplex were performed. Table 1 sets out their calculated CRF values as well as the peak asymmetry coefficients. The simplex was moved in the direction laid down by the rules of the sequential simplex method, including reflection, expansion and contraction vertices. All calculations were performed by hand using the simplex algorithm. ${ }^{19}$ The initial experimental points were selected according to the maximum CRF values obtained, from the best (vertex 2 with $\mathrm{CRF}=9.92$ ) to the worst (vertex 3 with $\mathrm{CRF}=$ -49.13). On the other hand, the shape of the chromatographic peaks at these points was rather poor; the coefficients of asymmetry ranged from 1.15 to 3.25 for vertex 2 and from 1.63 to 3.75 for vertex 3 . Better coefficients of asymmetry for the separated ionic liquid cations were noted in the case of vertex 1 with $\mathrm{CRF}=-21.15\left(f_{\mathrm{AS}}=0.59-2.14\right)$ and for vertex 4 with $\mathrm{CRF}=2.25\left(f_{\mathrm{AS}}=0.63-3.0\right)$. This is the effect of the higher concentration of methanol in the mobile phase as well as the lower $\mathrm{pH}$ at these points, resulting in a shorter time analysis and peaks of greater symmetry. It was found that in a mobile phase with an acidic $\mathrm{pH}$, the residual silanols of the stationary phase acquire a positive charge and are protonized, which reduces their interactions with ionic liquid cations; this is because of electrostatic repulsion forces, which produces chromatographic signals of a better shape. ${ }^{4,18}$

In the next step, the vertex with the worst response is reflected through the centroid of the simplex (vertex 3 ) and a new simplex is established. However, in our work, the obtained simplex point was rejected, since the values of the variables lay beyond the accepted range. The new vertex in the simplex algorithm was obtained by contraction. When this experiment was carried out, the resulting CRF was 5.06, indicating that the simplex was moving in the right direction. The variable size simplex algorithm was used, and the next step to be applied was reflection. The CRF was used to estimate each vertex of the simplex; the most unpropitious conditions, corresponding to the worst response, were rejected. Thereafter, the search continued for more favorable vertices. The simplex was halted after 34 experiments, since there was no further significant improvement towards maximization of the CRF value. Figure 1 shows the variation in $\mathrm{CRF}$ with the experiment number; it can be seen that an optimum response was already achieved at vertex 17 Although experiment number 17 had a high CRF value (10.45), 
Table 1 Simplex steps for the separation of a mixture of selected ionic liquid cations

\begin{tabular}{|c|c|c|c|c|c|}
\hline $\begin{array}{l}\text { Experiment } \\
\text { number }\end{array}$ & Vertex & $\underset{\%}{\mathrm{MeOH},}$ & $\begin{array}{l}\text { Phosphate buffer/ } \\
\mathrm{mM}\end{array}$ & $\mathrm{pH}$ & CRF \\
\hline 1 & I & 20 & 5.00 & 3.00 & -21.15 \\
\hline 2 & I & 10 & 15.00 & 3.50 & 9.92 \\
\hline 3 & I & 3 & 25.00 & 4.00 & -49.13 \\
\hline 4 & I & 15 & 10.00 & 3.75 & 2.25 \\
\hline 5 & $\mathrm{C}_{\mathrm{W}}$ & 9 & 17.50 & 3.71 & 5.06 \\
\hline 6 & $\mathrm{R}$ & 3 & 23.40 & 4.30 & -51.65 \\
\hline 7 & $\mathrm{C}_{\mathrm{W}}$ & 7 & 18.80 & 3.98 & -6.78 \\
\hline 8 & $\mathrm{R}$ & 16 & 9.60 & 3.32 & -4.60 \\
\hline 9 & $\mathrm{C}_{\mathrm{W}}$ & 14 & 11.90 & 3.49 & 0.12 \\
\hline 10 & $\mathrm{R}$ & 9 & 16.50 & 3.81 & 4.64 \\
\hline 11 & $\mathrm{C}_{\mathrm{R}}$ & 6 & 16.50 & 3.63 & -5.85 \\
\hline 12 & $\mathrm{R}$ & 13 & 12.95 & 3.71 & 3.33 \\
\hline 13 & $C_{W}$ & 11 & 14.78 & 3.69 & 7.98 \\
\hline 14 & $\mathrm{R}$ & 11 & 15.02 & 3.45 & 7.68 \\
\hline 15 & $C_{R}$ & 12 & 13.65 & 3.57 & 4.56 \\
\hline 16 & $\mathrm{R}$ & 9 & 16.21 & 3.53 & 6.88 \\
\hline 17 & $C_{W}$ & 10 & 15.57 & 3.54 & 10.45 \\
\hline 18 & E & 9 & 15.32 & 3.84 & 7.26 \\
\hline 19 & $\mathrm{R}$ & 12 & 14.92 & 3.32 & 4.85 \\
\hline 20 & $\mathrm{C}_{\mathrm{W}}$ & 11 & 15.02 & 3.45 & 7.68 \\
\hline 21 & $\mathrm{R}$ & 10 & 15.22 & 3.71 & 9.49 \\
\hline 22 & $\mathrm{R}$ & 9 & 15.86 & 3.47 & 9.43 \\
\hline 23 & $C_{W}$ & 9 & 15.59 & 3.53 & 8.74 \\
\hline 24 & $\mathrm{R}$ & 11 & 15.05 & 3.63 & 6.21 \\
\hline 25 & $C_{W}$ & 10 & 15.19 & 3.61 & 10.8 \\
\hline 26 & $\mathrm{R}$ & 10 & 15.28 & 3.43 & 9.65 \\
\hline 27 & $\mathrm{C}_{\mathrm{W}}$ & 10 & 15.27 & 3.50 & 10.79 \\
\hline 28 & $\mathrm{R}$ & 10 & 15.68 & 3.60 & 10.81 \\
\hline 29 & E & 10 & 15.00 & 3.63 & 9.40 \\
\hline 30 & $\mathrm{R}$ & 10 & 15.76 & 3.51 & 10.83 \\
\hline 31 & E & 10 & 16.08 & 3.71 & 10.79 \\
\hline 32 & $\mathrm{R}$ & 10 & 15.00 & 3.43 & 9.62 \\
\hline 33 & $\mathrm{C}_{\mathrm{W}}$ & 10 & 15.27 & 3.50 & 10.79 \\
\hline 34 & $\mathrm{R}$ & 10 & 15.81 & 3.64 & 9.53 \\
\hline
\end{tabular}

I, Initial vertex; $\mathrm{R}$, reflection vertex; $\mathrm{E}$, expansion vertex; $\mathrm{C}_{\mathrm{R}}$, contraction vertex on the reflection side; $\mathrm{C}_{\mathrm{W}}$, contraction vertex on the worst side.

this early high CRF value was considered to be fortuitous, because nearly the same set of conditions reappeared near vertices $25-34$. This return to the optimum value increases one's confidence that the method is rugged and efficient. Although the highest CRF value was obtained at vertex 30 (CRF $=10.83$ ), the coefficients of asymmetry at this point ranged from 1.06 to 3.18. The response at vertex 32 was found to be the best of all the points of the simplex, because both criteria applied, $\mathrm{CRF}$ and $f_{\mathrm{AS}}$, were the most favorable. The mobile phase at the point corresponding to the optimum (vertex 32) consisted of $10 \% \mathrm{MeOH}$ and $90 \% 15 \mathrm{mM} \mathrm{KK_{2 }} \mathrm{PO}_{4} / \mathrm{H}_{3} \mathrm{PO}_{4}$ with $\mathrm{pH} 3.43$. Applying a solvent mixture in this proportion permitted separation of all the critical pairs of ionic liquid cations: 1-ethyl3-methylimidazolium/1-ethyl-3-ethylimidazolium $\quad(R>1.5)$, 1-ethyl-3-ethylimidazolium/1-propyl-3-methylimidazolium $(R=0.90)$, 1-propyl-3-methylimidazolium/1-propyl-3-ethylimidazolium $(R>1.5)$, 1-butyl-3-methylimidazolium/ $N$-butyl-4methylpyridinium $(R>1.5)$ and $N$-butyl-4-methylpyridinium/1butyl-3-ethylimidazolium $(R=1.05)$. The coefficients of asymmetry for all of the analyzed compounds at the optimum of the simplex algorithm ranged from 0.83 to 2.91 , and the analysis time was $26.52 \mathrm{~min}$. Figures 2 and 3 present chromatograms

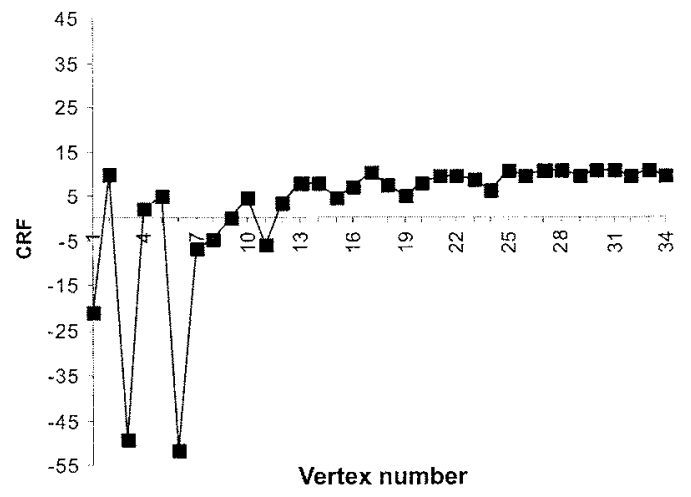

Fig. 1 Relationship between the chromatographic response function (CRF) and the experiment number during simplex optimization.

obtained under the initial experimental condition (vertex 1) and the optimized condition (vertex 32).

Methanol as well as the buffer concentration can significantly vary the retention and the resolution of the cationic analytes. However, the $\mathrm{pH}$ of the eluent seems to be less important concerning resolution development. Thus, the cation $\left(\mathrm{K}^{+}\right)$ content in the eluent controls the retention; therefore, cation exchange or cation exclusion should work with this polar reverse stationary phase.

\section{Conclusions}

The sequential simplex method was applied to optimize the separation of a congeneric group of imidazolium ionic liquid cations and one alkylpyridinium cation. A chromatographic response function (CRF), which included the number of peaks, the resolution between adjacent peaks, a specified analysis time and the individual retention times relative to a minimum retention time, was calculated to evaluate the quality of the individual chromatograms.

The peak shapes of all the analyzed ionic liquid cations, evaluated with the aid of the coefficient of asymmetry, were also taken into account in the optimization process. In total 34 experiments were carried out to optimize a three-variable system comprising methanol and an aqueous solution of phosphate buffer of appropriate $\mathrm{pH}$. The mobile phase, consisting of $10 \%$ $\mathrm{MeOH}$ and $90 \% 15 \mathrm{mM} \mathrm{KH} \mathrm{PO}_{4} / \mathrm{H}_{3} \mathrm{PO}_{4}$ with $\mathrm{pH} 3.43$, had an optimal composition of eluents and permitted good resolution $(R=0.9->1.5)$ of all five critical pairs: 1-ethyl-3methylimidazolium/1-ethyl-3-ethylimidazolium, 1-ethyl-3ethylimidazolium/1-propyl-3-methylimidazolium, 1-propyl-3methylimidazolium/1-propyl-3-ethylimidazolium, 1-butyl-3methylimidazolium/ $N$-butyl-4-methylpyridinium and $N$-butyl-4methylpyridinium/1-butyl-3-ethylimidazolium; it also yielded good peak shapes $\left(f_{\mathrm{AS}}=0.83-2.91\right)$ and an analysis time of $26.52 \mathrm{~min}$. A conceptually simple and useful tool for developing a method for analyzing imidazolium and pyridinium ionic liquids, the variable-size simplex algorithm, was found to be straightforward to implement.

\section{Acknowledgements}

Financial support was provided by the Polish Ministry of Education and Research under grants 2P04G 083 29, 2P04G 11829 and DS 8200-0085-8. The grant of the University of Gdańsk nr BW 8000-5-0138-8 is greatly acknowledged. 


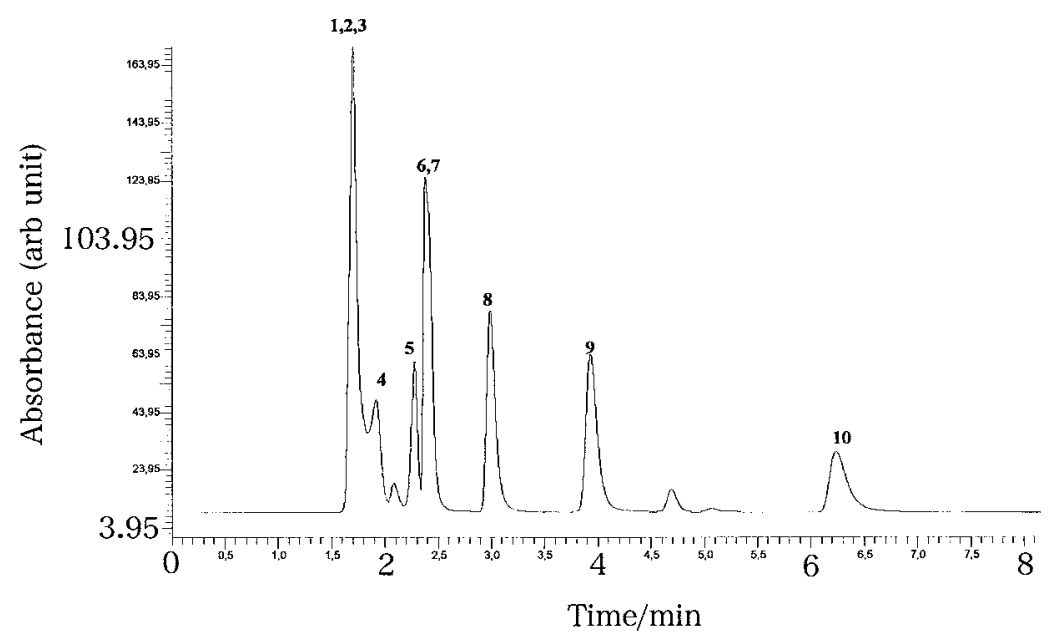

Fig. 2 Chromatogram of an ionic liquid cations mixture obtained under the initial conditions (vertex number 1). The peaks correspond to: 1, EMIM; 2, EEIM; 3, PMIM; 4, PEIM; 5, BMIM; 6, MBPy; 7, BEIM; 8, BzMIM; 9, EBzMIM; 10, HMIM.

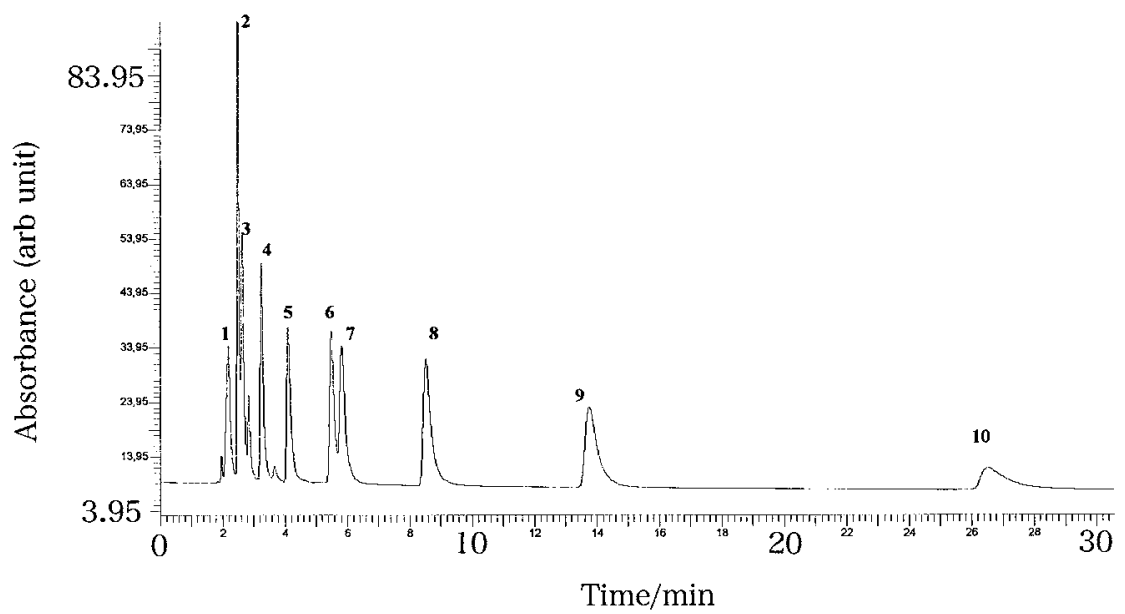

Fig. 3 Chromatogram of an ionic liquid cations mixture obtained under optimum conditions (vertex 32). The peak numbers are the same as those in Fig. 2.

\section{References}

1. P. Wasserscheid and T. Welton, "Ionic Liquids in Synthesis", 2003, Wiley VGH, Weinheim.

2. P. Stepnowski, A. Müller, P. Behrend, J. Ranke, J. Hoffmann, and B. Jastorff, J. Chromatogr., A, 2003, 993, 173.

3. P. Stepnowski, Anal. Bioanal. Chem., 2005, 381, 189.

4. P. Stepnowski and W. Mrozik, J. Sep. Sci., 2005, 28, 149.

5. M. J. Markuszewski, P. Stepnowski, and M. P. Marszałł, Electrophoresis, 2004, 25, 3450.

6. P. Stepnowski, J. Nichthauser, W. Mrozik, and B. Buszewski, Anal. Bioanal. Chem., 2006, 385, 1483.

7. J. Nichthauser and P. Stepnowski, J. Chromatogr. Sci., in press.

8. W. Spendley, G. R. Hext, and F. R. Himsworth, Technometrics, 1962, 4, 441.

9. K. W. C. Burton and G. Nickless, Chemom. Intell. Lab.
Syst., 1987, 1, 135 .

10. C. Różycki, Chem. Anal., 1993, 38, 681.

11. T. Öberg, Chemom. Intell. Lab. Syst., 1998, 44, 147.

12. J. A. Nelder and R. Mead, Comput. J., 1965, 308.

13. A. Gustavsson and J. E. Sundkvist, Anal. Chim. Acta, 1985 , $167,1$.

14. L. R. Parker Jr., M. R. Cave, and R. M. Barnes, Anal. Chim. Acta, 1985, 175, 231.

15. D. Betteridge, A. P. Wade, and A. G. Howard, Talanta, 1985, 32, 709 .

16. D. Betteridge, A. P. Wade, and A. G. Howard, Talanta, 1985, 32, 723.

17. J. C. Berridge, J. Chromatogr., 1989, 485, 3.

18. S. Kowalska, B. Buszewski, and P. Stepnowski, J. Sep. Sci., 2006, 29, 1116

19. A. Wasik, B. Radke, J. Bolałek, and J. Namieśnik, Chemosphere, 2007, 6, 1. 